sous forme de sels, est au point de vue physiologique d'une très grande importance et donne la possibilité d'alimenter le bétail uniquement avec du fourrage conservé par les acides, sans dérangement quelconque.

(A suivre).

\title{
SUR LA THÉORIE DE LA POLYMÉRISATION DE LA CASÉINE.
}

\author{
par MaURICE BEAU \\ Ingénieur agronome.
}

Nous avons exposé dans un précédent article (1) une nouvelle théorie permettant de rendre compte d'une façon simple des phénomènes qui se passent dans la coagulation de la caséine par la présure, et dans la transformation de la caséine en paracaséine.

Cette théorie consiste en résumé à dire :

$1^{0}$ que, dans la easéine telle qu'elle sa trouve dans le lait, il y a liaison entre un certain nombre de groupes $\mathrm{COOH}$ d'une molécule et un nombre égal de groupes $\mathrm{NH}^{2}$ d'une autre molécule, d'après un schéma analogue au suivant :

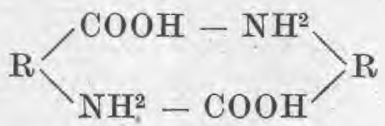

(Ce sont probablement ces liaisons d'ordre chimique qui, se répétant sur un nombre considérable de molécules, arrivent à constituer les agrégats physiques qui, sous le nom de micelles de caséine, sont visibles à l'ultra-microscope.)

$2^{\circ}$ que l'action de la présure consiste d'abord à seinder ces liaisons, de manière à rendre de nouveau libres les groupements $\mathrm{COOH}$ et $\mathrm{NH}^{2}$, qui peuvent alors se combiner avec les radicaux $\mathrm{CaO}$ et $\mathrm{P}^{2} \mathrm{O}^{5}$ présents dans le liquide, pour donner le phosphoeaséinate de chaux en agrégats physiques de plus en plus volumineux et polymérisés, qui constituent le bloc de caillé, et qui donnent après séchage la caséine dite à la présure.

Si cette théorie est exacte, il en résulte que le premier effet de la présure doit être d'augmenter simultanément l'acidité et l'alcalinité de la caséine, par suite de la mise en liberté, à la fois, de valences $\mathrm{COOH}$ et $\mathrm{NH}^{2}$. Mais il va de soi qu'il n'est pas facile de mettre ce résultat en évidence; en effet, si on se borne à titrer à la manière ordinaire l'acidité moléculaire totale ou potentielle de la easéine, surtout en opérant avec des acides ou des alcalis forts, on ne trouve aucune différence, la caséine et la paracaséine se comportant dans ce cas comme des corps entièrement dépolymérisés.

(1) Le Lait, juillet-août 1932. 
Mais peut-être est-il possible d'observer une différence en mesurant l'acidité ionique ou actuelle de la caséine, c'est-à-dire en déterminant le $p \mathrm{H}$, d'une part, sur la caséine primitive et, d'autre part, sur la même caséine modifiée par la présure, sur la paracaséine ; il faut seulement, pour cette dernière, éviter la recombinaison des groupements $\mathrm{COOH}$ et $\mathrm{NH}^{2}$ avec la chaux et l'acide phosphorique présents.

C'est ce qu'ont fait deux auteurs américains : PaLmer et Richardson, dans un travail publié il y a quelques années (1), mais dont nous n'avons eu que récemment l'original entre les mains. Ces auteurs ont d'abord eonstaté que la caséine et la paracaséine isoélectriques (pures) ne peuvent être différenciées chimiquement, soit par l'analyse élémentaire, soit par la distribution des acides aminés, soit par les méthodes de racémisation. Ils ont alors cherché une différence en opérant comme suit :

Du lait écrémé de centrifuge est chauffé, puis passé par une supercentrifuge Sharples, de façon à enlever toute trace de matière grasse ; ce lait est ensuite dialysé dans des sacs de collodion en vue d'éliminer tous les sels solubles, en particulier la chaux et l'acide phosphorique en solution; enfin, on dilue avec un égal volume d'eau, et on ajoute à $30^{\circ} \mathrm{C}$. un excès d'extrait de présure.

On maintient au contact pendant une demi-heure, sans qu'il se produise de coagulation, puis on isole, d'une part, la paracaséine pure en partant de ce liquide et, d'autre part, la caséine pure en partant du lait primitif, le tout par la méthode de précipitation à l'acide indiquée par L. L. van Styke (2).

En somme, on fait agir la présure directement sur la caséine en l'absence de chaux et d'acide phosphorique solubles, c'est-à-dire dans des conditions telles que la coagulation ne peut se produire (et elle ne se produit effectivement pas), et que le phosphocaséinate de chaux ne peut se former.

On mesure ensuite, sur chacun des deux produits, caséine et paracaséine, le $p H$ des liqueurs obtenues en délayant $1 \mathrm{gr}$. du corps dans $100 \mathrm{~cm}^{3}$ d'eau et en ajoutant des multiples de $0 \mathrm{~cm}^{3} 1$, soit d'un réactif alcalin (solution normale de soude ou de chaux), soit d'un réactif acide (solution standard de $\mathrm{HCl}$ ).

Dans ces conditions, la caséine et la paracaséine entrent en liaison avec l'alcali ou l'acide, mais les quantités d'alcali ou d'acide respectivement neutralisées doivent être plus grandes pour la paracaséine que pour la caséine, à cause de la mise en liberté par la présure des valences $\mathrm{COOH}$ et $\mathrm{NH}^{2}$.

(1) L. S. Palmer et G. A. Richardson. The colloid chemistry of rennet coagulation New-York, 1925.

(2) Voir la description de cette méthode dans notre ouvrage sur "La Caséine ". Paris 1932. Editions de la Revue Le Lait. 
Autrement dit, pour obtenir le même $p \mathrm{H}$, il doit falloir employer pour la paracaséine plus d'alcali ou plus d'acide que pour la caséine ; c'est bien en effet ce que Palmer et Richarbson ont constaté, comme le montre le tableau suivant, extrait de ceux publiés par ces auteurs.

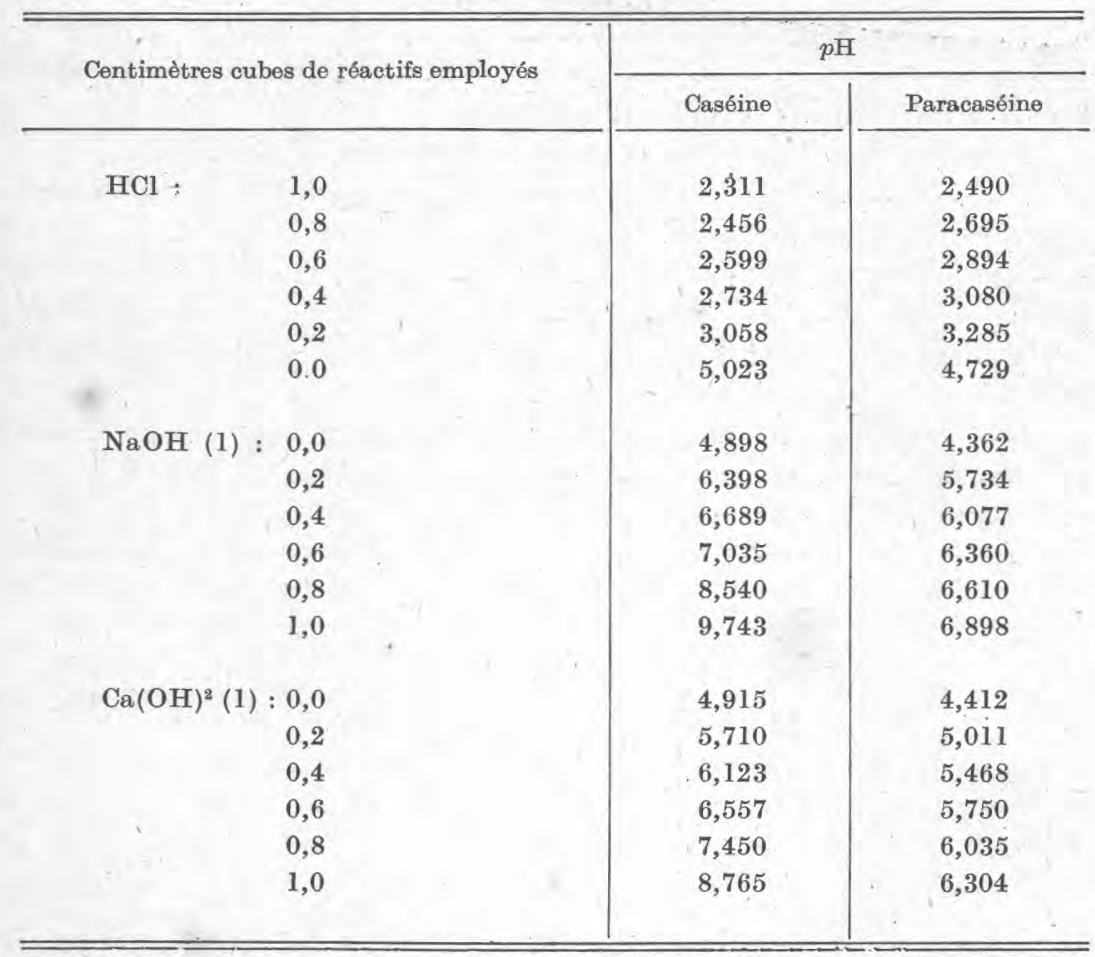

(1) Les auteurs ont poussé les quantités de réactifs alcalins jusqu’à $3 \mathrm{~cm}^{3}$; les résultats restent les mêmes.

Les chiffres précédents montrent :

$1^{\circ}$ que la paracaséine pure présente toujours un $p H$ inférieur à celui de la caséine pure correspondante. Ce résultat s'explique facilement dans notre hypothèse : on sait que la molécule de caséine (2) renferme un plus grand nombre de groupes acides (carboxyles) que de groupes alcalins (amines); rien d'étonnant par suite qu'un plus grand nombre de groupes acides soient mis en liberté, ce qui donne un excès d'acidité à la molécule, c'est-à-dire un $p \mathrm{H}$ moindre;

$2^{\circ}$ qu'il faut plus de réactif, quel qu'il soit (acide ou alcalin), pour obtenir la même acidité ou la même alcalinité avec la paracaséine qu'avec la caséine, l'excédent de réactif employé provenant

(2) Voir notre ouvrage sur "La Caséine". 
de l'excédent des valences acides ou alcalines libérées par la présure, et étant sans doute, au moins grosso modo, proportionnel à cet excédent;

$3^{0}$ qu'au voisinage du $p \mathbf{H}$ égal à 6,6-6,7, e'est-à-dire du $p \mathbf{H}$ du lait frais, il faut environ le double d'alcali pour obtenir le même $p H$ avec la paracaséine qu'avec la caséine, ce qui explique pourquoi L. L. van SLYke considérait que le poids moléculaire de la caséine n'était que la moitié de celui de la caséine ;

$4^{\circ} q u^{\prime} e n$ dehors $d u$ point isoélectrique $(p H=4,6-4,7)$, surtout pour des quantités élevées de réactifs, il y a à peu près parallélisme entre les courbes de saturation de la caséine et celles de la paracaséine, obtenues en portant en abscisses les $p H$ et en ordonnées les quantités de réactifs, c'est-à-dire que les excédents de réactifs nécessaires pour obtenir le même $p H$ avec la paracaséine comparée à la caséine sont à peu près constants.

Dans l'expérience de Palmer et Richardson, ces excédents ont été les suivants, comptés par gramme de caséine et exprimés en molécules-grammes $\times 10^{-5}$.

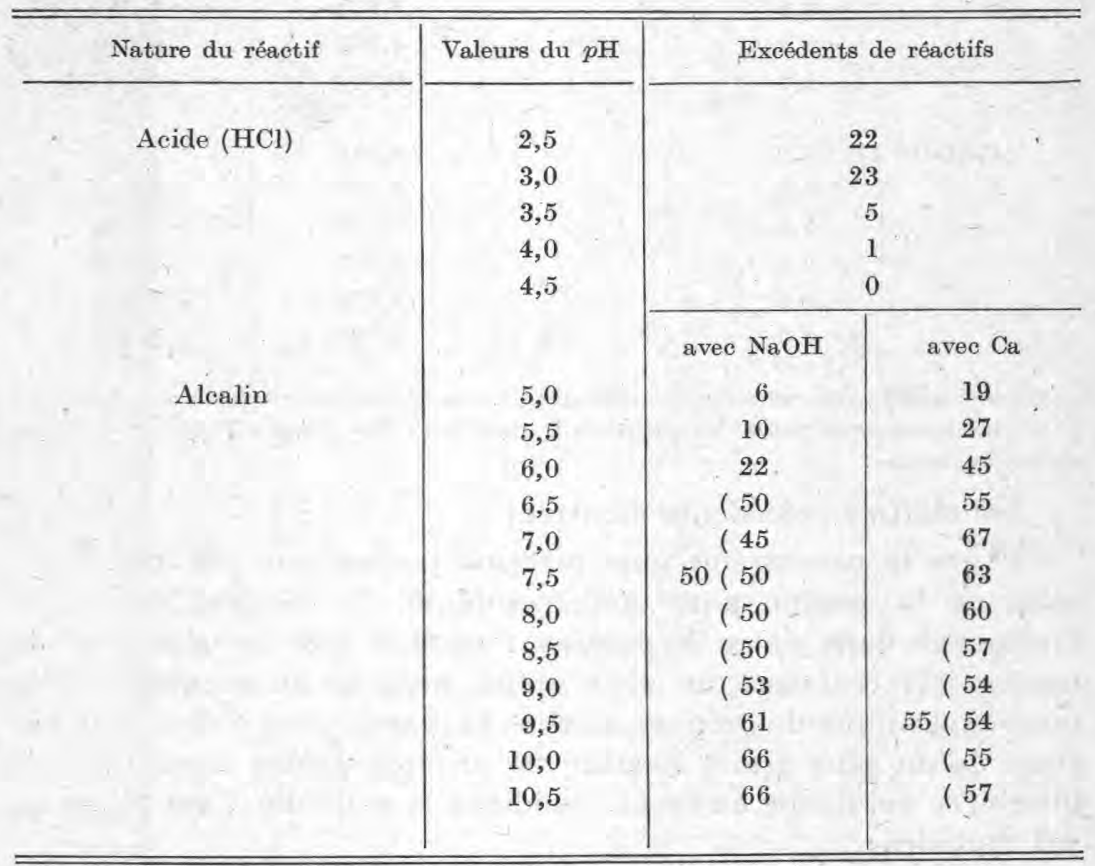

Si l'on met de côté ce qui se passe au voisinage du point isoélectrique, c'est-à-dire pour des $p \mathrm{H}$ compris entre $3,5(\mathrm{HCl})$ et $6,0(\mathrm{NaOH})$, où la saturation èst évidemment très incomplète, et l'attaque des grains de caséine par le réactif difficile à cause du peu 
de concentration de ce dernier, on constate qu'il y a ensuite, aussi bien pour l'acide que pour l'alcali, une constance remarquable des excédents (1).

Si ces excédents mesurent le degré de l'action de la présure sur la molécule de caséine, cette action serait représentée en moyenne par $50 \times 10^{-5}$ molécules-grammes de soude, soit $20 \mathrm{mgr}$. de soude $\mathrm{NaOH}$ par gramme de paracaséine.

Avec la chaux, la constance des excédents n'est obtenue que pour un $p \mathbf{H}$ supérieur à 8 , et l'action de la présure serait représentée par $55 \times 10^{-5}$ molécules-grammes de chaux, soit encore $20 \mathrm{mgr}$. de ehaux $\mathrm{Ca}(\mathrm{OH})^{2}$ par gramme de paracaséine.

Il est remarquable qu'il faille pour cela autant de chaux que de soude en poids, alors que d'a près les rapports des poids moléculaires : $\frac{2 \mathrm{NaOH}}{\mathrm{Ca}(\mathrm{OH})^{2}}=\frac{80}{74}$, il devrait falloir un peu moins de chaux que de soude; mais cela provient probablement de ce que la chaux est une base plus faible que la soude, et ce fait peut expliquer aussi que la constance des excédents débute à un $p \mathrm{H}$ plus élevé.

Tout ce qui précède montre d'ailleurs que l'ensemble de ces opérations de titrage est extrêmement complexe et que le résultat dépend de divers facteurs, dont les deux principaux sont les suivants :

$1^{\circ}$ l'adsorption physique du réactif par les micelles de la caséine et de la paracaséine (c'est à ce facteur que HofFmann et GorTner (2) attribuent les résultats obtenus pour des $p H$ inférieurs à 2,5 ou supérieurs à 10,5 ; dans ce dernier eas, toutefois, il y a lieu de tenir compte aussi de l'attaque chimique de la caséine par l'alcali);

$2^{\circ}$ la combinaison chimique du réactif avec les valences disponibles de la caséine ou de la paracaséine; mais ces valences sont disponibles sous deux formes:

a) ou bien elles sont libres, ce qui, dans la molécule de caséine, est le cas pour les 8 à $16 \mathrm{COOH}$ reconnus tels par les auteurs américains (3), auxquels il faut ajouter pour la paraeaséine les valences libérées par la présure ;

b) ou bien elles sont libérables par la réaction elle-même, ce qui est le cas à la fois dans la caséine et dans la paracaséine, pour la plupart des valences acides ou alcalines présentes, notamment sous forme d'acides ąmidés.

Dans ce cas, le résultat dépend de la force du réactif; on sait en

(1) Au-dessus du pH 8,5, il y a sans doute avec la soude commencement de décomposition de la caséine par l'alcali.

(2) W. F. Hoffmann et R. A. Gortaner. Colloid symposium monograph, Ch. 20. New-York, 1925.

(3) Voir notre ouvrage sur \& La Caséine ". 
effet que l'acide chlorhydrique ou la soude concentrés décomposent la caséine en une série de corps amidés plus ou moins "dégradés" aboutissant pour finir à l'acide carbonique et à l'ammoniaque.

A faibles doses, les réactifs sont aussi susceptibles, suivant leur "force ", de donner des résultats différents, comme nous venons de le voir pour la chaux et la soude.

Quoi qu'il en soit, Palmer et Riohardson eoncluent comme suit :

"Il est évident que la présure, agissant sur un caséinate de calcium incomplètement formé, en dispersion colloïdale, au $p \mathrm{H}$ du lait, le transforme en un paracaséinate de calcium encore moins complètement formé (1), et dont le pouvoir de liaison ohimique, à la fois avec les bases et les acides est modifié de façon permanente. La nature du réarrangement moléculaire ou du changement superficiel "(des micelles) " qui produit cette augmentation de la capacité de liaison, n'est pas claire, mais elle ne trouve certainement pas son explication dans le principe d'une simple division moléculaire ou peptisation. Ni l'une ni l'autre de ces dernières modifications. ne peut seule augmenter "(à la fois) ) les quantités d'alcali et d'acide combinées par gramme de caséine. Il est évident que l'instabilité du paracaséinate faiblement saturé, accerue par les hautes températures employées dans la coagulation par la présure, est responsable de sa plus grande sensibilité vis-à-vis des cations, et explique sa coagulation."

Cette conclusion rejoint celle que nous avons donnée dans la théorie de la polymérisation : la easéine, légèrement polymérisée suivant le schéma [1], est dépolymérisée tout d'abord par la présure, d'où formation d'un corps moins saturé que la easéine primitive, lequel, en présence de chaux et d'acide phosphorique se repolymérise suivant les schémas que nous avons indiqués dans notre précédent article. La polymérisation de la caséine, d'instable qu'elle était, devient stable, et le produit obtenu est la caséine présure du commerce, toujours colloïdale, hautement polymérisée, très stable et plastique.

L'opération, qui consiste en une transposition de liaisons de saturation et en leur consolidation, est essentiellement le fait d'une action catalytique de polymérisation physico-chimique.

(1) On pourrait aussi bien dire que la présure, agissant sur là caséine incomplètement saturée, la transforme en paracaséine encore moins complètement saturée, car c'est là en réalité ce qui se passe dansi'expérience de PaLmer et RioHardson. 